Recherches en didactique des langues et des cultures

Les cahiers de l'Acedle

13-3 | 2016

Travailler sur et à partir de textes écrits en classe de langue étrangère

De phrases illustrant la grammaire au texte porteur de sens : quelques réflexions sur des manuels de latin

\title{
Antje-Marianne Kolde
}

\section{(2) OpenEdition}

\section{Journals}

Édition électronique

URL : http://journals.openedition.org/rdlc/1432

DOI : $10.4000 /$ rdlc. 1432

ISSN : 1958-5772

Éditeur

ACEDLE

Référence électronique

Antje-Marianne Kolde, «De phrases illustrant la grammaire au texte porteur de sens : quelques

réflexions sur des manuels de latin », Recherches en didactique des langues et des cultures [En ligne],

13-3 | 2016, mis en ligne le 31 décembre 2016, consulté le 19 avril 2019. URL : http://

journals.openedition.org/rdlc/1432 ; DOI : 10.4000/rdlc. 1432

Ce document a été généré automatiquement le 19 avril 2019.

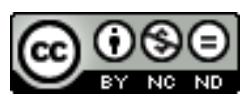

Recherches en didactique des langues et des cultures is licensed under a Creative Commons AttributionNonCommercial-NoDerivatives 4.0 International License 


\title{
De phrases illustrant la grammaire au texte porteur de sens : quelques réflexions sur des manuels de latin
}

\author{
Antje-Marianne Kolde
}

\section{Introduction : du focus « grammaire " au focus « communication »}

1 Comment apprendre une langue ? Comment enseigner une langue ? Convient-il de se focaliser prioritairement sur le fonctionnement d'une langue ou sur son utilisation dans un cadre le moins artificiel possible? Voilà bien des questions aussi vieilles que les langues elles-mêmes et leur enseignement et apprentissage. Les réponses que l'on peut proposer de leur donner sont multiples et varient selon l'époque et le pays considérés, selon la tradition nationale et régionale de l'enseignement des langues et, finalement, selon la langue en question.

De fait, dès la fin du XIXe siècle, l'enseignement/apprentissage des langues modernes s'est peu à peu éloigné d'une approche centrée sur la grammaire et le lexique pour se tourner par le biais de la méthode directe ou d'approches audio-orales et audio-visuelles vers la réception et la production orales (cf. Martinez, qui donne un bon aperçu des diverses méthodologies dans son chapitre II) : dès lors, en accord avec l'approche actionnelle, « on part du principe que l'apprenant de langue est en train de devenir un usager de la langue » (CECR, p.40) et on focalise sur la communication en langue-cible.

Qu'en est-il de l'autre groupe de langues enseignées à l'école, celui des langues classiques, le latin et le grec? Puisqu'on ne les acquiert pas afin de communiquer par leur biais comme on communique en anglais par exemple, l'enseignement des langues classiques peut-il avoir d'autres objectifs principaux que leur lexique et leur fonctionnement grammatical ? En d'autres termes, sa cible peut-elle changer, passant d'une perspective centrée sur la description de la langue à une perspective axée sur l'utilisation de la langue en tant que moyen d'accès à des textes, et cela depuis le début? 
4 Pour tenter de répondre à cette question, il convient de préciser dans un premier temps quelles activités langagières constituent un objectif tant de l'enseignement des langues classiques que de celui des langues modernes et peuvent donc contribuer à déterminer si l'enseignement/apprentissage des langues classiques a suivi une évolution semblable à celui des langues vivantes. Nous verrons qu'il s'agit de diverses activités en relation avec des textes. Ensuite seront analysés cinq moyens d'enseignement de latin utilisés dans

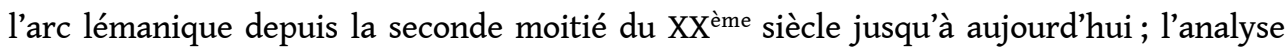
portera sur le rôle que le ou les auteur(s) du manuel attribue(nt) au texte écrit : sert-il à illustrer un point de grammaire préalablement expliqué ou est-ce son contenu, l'information ou le message qu'il contient, qui est mis en avant?

\section{Les activités langagières autour du texte : en langues classiques comme en langues modernes}

5 Les langues servent en premier lieu à communiquer. Il s'ensuit qu' « afin de réaliser des tâches de communication, les usagers de la langue doivent s'impliquer dans des activités langagières communicatives. [...] Dans ces cas, l'événement communicatif peut être considéré comme parler, écrire, écouter ou lire un texte » (CECR, p.48). Produit par le Conseil de l'Europe pour l'Europe moderne, le CECR est consacré à l'apprentissage, à l'enseignement et à l'évaluation des langues vivantes et ne fait pas cas des langues classiques enseignées à l'école. Le latin et le grec ne comptant plus de locuteurs natifs, lire un texte est la seule parmi les quatre activités langagières susmentionnées à constituer un objectif tant de l'enseignement/apprentissage des langues classiques que de celui des langues modernes. L'école transmettant une culture générale, l'enseignement de spécialiste qu'est le thème est réservé à l'université.

6 Selon le mode dans lequel la communication a lieu, les activités langagières relèvent « de la réception, de la production, de l'interaction, de la médiation» (CECR, p.18). La lecture d'un texte donne lieu à deux modes de communication communs aux deux groupes de langues: la réception et la médiation. Cette dernière consiste en diverses activités: "Parmi les activités de médiation écrite on trouve, par exemple : traduction précise [...]; traduction littéraire [...]; résumé de l'essentiel [...] en $L 2$ ou entre $L 1$ et $L 2$; reformulation [....]» ( CECR, p.71). Si dans le cadre des langues modernes, la médiation peut être orale et/ou écrite, dans le cadre des langues classiques, la composante écrite est omniprésente, puisque le point de départ est toujours constitué d'un texte écrit. Plus précisément, comme il ressort de la citation, lorsque le texte source est en L2, la traduction est formulée en $\mathrm{L} 1$, le résumé ou la reformulation en $\mathrm{L} 1$ ou en $\mathrm{L} 2$ - voilà bien ce qui se passe en cours de langues classiques, à ceci près que le résumé ou la reformulation sont formulés en L1, puisque on ne procède qu'à des activités de réception. Il s'ensuit que les activités de médiation en latin ou en grec impliquent le recours à deux langues différentes : le latin ou le grec pour la réception et le français pour la production.

7 Le fait que les deux groupes de langues partagent plusieurs objectifs d'enseignement/ apprentissage ressort aussi d'un document rédigé par la Division des Politiques linguistiques du conseil de l'Europe ${ }^{1}$ et consacré à l'enseignement des langues étrangères en général, tant classiques que vivantes : «Qu'il s'agisse de langues vivantes ou classiques, leur enseignement est axé sur la connaissance et la compréhension de la nature de la langue, d'autres cultures et de la culture de l'apprenant» (COE, s.d: 4). Les ressemblances entre langues 
modernes et langues classiques côtoient cependant les différences, et cela au niveau des objectifs relatifs aux compétences linguistiques. En effet, au niveau des compétences linguistiques, l'enseignement/apprentissage des langues modernes vise des objectifs fonctionnels, qui résident dans des compétences tant réceptives que productives, tant orales qu'écrites, utiles pour la communication avec les locuteurs de la langue-cible et cela à diverses fins, comme la négociation, le travail ou les loisirs. Quant à l'enseignement/apprentissage des langues classiques, il ne vise que la compétence réceptive dans la langue-cible, toute production étant réalisée dans la langue de scolarisation. (COE, s.d : 4)

8 Étant donné que la lecture de textes et la médiation constituent toutes deux des modes de communication langagière auxquels on recourt en classe de langues anciennes, analyser la place que les moyens d'enseignement octroient aux textes et aux activités en lien avec eux permet de répondre à la question initiale, relative à la présence ou à l'absence d'un changement de paradigme dans l'enseignement/apprentissage des langues classiques. ${ }^{2}$

Précisons encore que pour l'acception de " texte", on suit la définition donnée par le CECR : "Est définie comme texte toute séquence discursive (orale et/ou écrite) inscrite dans un domaine particulier et donnant lieu, comme objet ou comme visée, comme produit ou comme processus, à activité langagière au cours de la réalisation d'une tâche» (CECR, p.15).

\section{Corpus des moyens d'enseignement et points examinés}

10 Avant d'analyser la place octroyée aux textes et les activités langagières proposées, il convient de définir le corpus des manuels retenus et de préciser les questions à la lumière desquelles on examinera les manuels en question.

\section{Présentation des moyens d'enseignement : le corpus}

11 Afin de conserver une certaine unité, la présente recherche se limite aux manuels prescrits pour l'enseignement du latin en Suisse romande, plus précisément dans les cantons de Genève et de Vaud ; le public-cible est celui du secondaire 1, autrement dit des élèves de 12 à 15 ans. Pour garantir une certaine profondeur chronologique, nous avons retenu cinq moyens d'enseignement dont le plus ancien a été utilisé dès le milieu du XX ème siècle et le plus récent est en cours d'utilisation. Un tableau récapitulatif succinct complètera la présentation des manuels, désignés sous les noms consacrés par l'usage des enseignants et des élèves, mêlant noms d'auteurs, de séries, d'éditeurs et titres.

Le plus ancien est le " Debeauvais », un manuel français utilisé dans le Canton de Vaud en tout cas depuis le milieu du XX⿳亠㐅⿵冂⿰⿱丶㇀⿱㇒丶⿱一土⿵冂丶 quand précisément le «Debeauvais » a été introduit dans l'enseignement vaudois. Bien qu'il compte de nombreux volumes déclinant années d'enseignement et activités diverses, ${ }^{3}$ les enseignants du canton de Vaud n'en ont utilisé que quatre : la "grammaire ", 1 ère

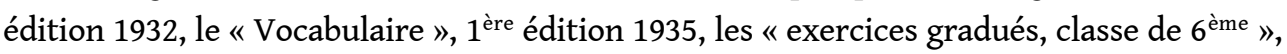
$1^{\text {ère }}$ édition 1953, et les « exercices gradués, classe de de $5^{\text {ème }}$ ", $1^{\text {ère }}$ édition 1955. Ces livres ont subi de nombreuses rééditions, au cours desquelles le programme a été allégé et les exercices simplifiés. 
13 Puis vient le « Godel », un manuel genevois utilisé à Genève de 1966 à 1986, formé de deux volumes, $1^{\text {ère }}$ édition 1966 pour le premier et 1969 pour le second. Les deux volumes se suivent, le second débutant là où le premier s'arrête.

Ces deux manuels, le « Debeauvais » et le « Godel », sont remplacés dans les deux cantons en même temps, en 1992, par le "Latinissime ", une adaptation romande du manuel français « Salvete », 1 ère édition 1988 ; ce manuel est aussi composé de deux volumes, dont le second commence par des révisions.

Lorsqu'en 2000, l'adaptation romande "Latinissime » est épuisée, le canton de Genève opte pour l'édition française d'origine, le « Salvete». Les deux volumes du manuel ont été complétés par deux brochures d'exercices par année d'apprentissage.

Dans le canton de Vaud par contre, on remplace le «Latinissime " par le « Magnard », lui aussi un livre français, 1ère édition 1997 pour le premier volume, 1998 pour le deuxième et 1999 pour le troisième, repris sans adaptation.

En 2012, le «Latinissime » dans le canton de Genève et le « Magnard » dans le canton de Vaud sont remplacés par le « Latin Forum ». Ce manuel, composé de trois volumes, datant respectivement de 2012, 2013 et 2014, est une adaptation du « Magnard » à la Romandie et au Plan d'Études Romand (PER). Les volumes 2 et 3 commencent par des chapitres de révision.

Les cinq manuels sommairement présentés débutent par un chapitre consacré à un rappel de grammaire française et/ou de l'écriture de la langue latine, donnant pour la plupart d'entre eux un bref aperçu de l'évolution linguistique aboutissant au français.

\begin{tabular}{|l|l|l|l|l|}
\hline Nom & 1 ère édition & Lieu de parution & Dates d'utilisation & Canton(s) \\
\hline « Debeauvais " & $1932 ; 1935 ; 1953 ; 1955$ & Paris & $?-1992$ & Vaud \\
\hline « Godel » & $1966 ; 1969$ & Genève & $1966-1992$ & Genève \\
\hline « Latinissime » & $1992 ; 1993$ & Lausanne & $1992-2000$ & Genève et Vaud \\
\hline « Salvete " & $1988 ; 1989$ & Paris & $2000-2012$ & Genève \\
\hline « Magnard & $1997 ; 1998 ; 1999$ & Paris & $2000-2012$ & Vaud \\
\hline « Latin Forum " & $2012 ; 2013 ; 2014$ & Lausanne & $2012-$ & Vaud et Genève \\
\hline
\end{tabular}

\section{Points examinés}

Pour déterminer la place que ces manuels octroient aux textes et les activités langagières qu'ils proposent en lien avec les textes, j'analyserai ces manuels à l'aide de diverses questions, qui doivent remplir deux critères. D'une part, elles doivent permettre d'examiner des manuels très différents, séparés les uns des autres par une grande distance temporelle et issus de systèmes scolaires divers, reflétant donc des méthodologies et des approches diverses. D'autre part, elles doivent être pertinentes par rapport à la perspective considérée, à savoir les activités langagières proposées et les modes de communication réalisés. 
20 En fonction de ces deux critères, on a retenu sept questions, deux (1 et 2) qui portent sur les caractéristiques " physiques » des textes et cinq (3-7) qui concernent l'utilisation que les manuels en proposent :

1. Les chapitres du moyen d'enseignement comptent-ils un (des) texte(s)?

2. S'agit-il de texte(s) entièrement fabriqué(s), appuyé(s) sur des textes authentiques ou authentique(s)?

3. À quel moment du chapitre recourt-on au(x) texte(s)?

4. Existe-t-il un lien entre les textes des diverses leçons?

5. Existe-t-il un lien entre le(s) texte(s) et le(s) sujet(s) de grammaire de la leçon ?

6. Existe-t-il des liens nets entre le(s) texte(s) et le vocabulaire de la leçon ?

7. Le manuel pose-t-il des questions de compréhension sur le(s) texte(s)?

\section{Moyens d'enseignement et textes}

\section{Les chapitres du moyen d'enseignement comptent-ils un (des) texte}

(s)?

21 Les livres d'« exercices gradués » du « Debeauvais » ne sont pas divisés en chapitres ou leçons numérotés. Ils sont structurés selon des points de grammaire - par exemple «phrases complétives ». Axés sur l'acquisition lexicale et grammaticale, ils procèdent essentiellement par la traduction de phrases du français en latin (thème) et du latin en français (version). Parfois, les phrases de version sont regroupées en petits textes, insérés parmi les exercices composés de phrases dépourvues de lien sémantique les unes avec les autres.

Le « Godel » est divisé en leçons regroupant chacune un exposé grammatical, une liste de mots à apprendre et des exercices d'application. Ces exercices proposent également la traduction de phrases isolées en thème et en version. À la leçon 17 se trouve le premier texte suivi ; à partir de là, un texte suivi clôt chaque leçon.

Chaque leçon $\mathrm{du}$ "Latinissime » s'ouvre sur un petit texte latin constituant un tout cohérent, souvent de forme dialoguée. Les exercices de traduction, tant des thèmes que des versions, sont composés de phrases sans lien les unes avec les autres. Toutes les cinq leçons apparait une "étape" qui récapitule le vocabulaire appris lors des leçons précédentes et offre des exercices supplémentaires; ce n'est qu'à la troisième de ces étapes, donc après la leçon 15 , que l'on trouve le premier texte suivi que les élèves doivent traduire. Dans les brochures d'exercices genevoises par contre, le premier texte suivi, également donné en exercice de version, apparait à la leçon 5 ; dès lors, un texte suivi clôt la série d'exercices proposés pour chaque leçon.

Dans le "Magnard», chaque chapitre, y compris le premier, commence par un texte donné en bilingue suivi de deux autres textes que les élèves doivent traduire en version. En plus de cela, sont proposés divers exercices de traduction, thèmes et versions, composés de phrases isolées ; généralement, chaque chapitre se clôt par un thème et une version suivis, traitant de sujets divers, notamment de vie quotidienne, de mythologie et d'histoire.

Le «Latin Forum », finalement, reprend la structure du « Magnard »: chaque chapitre s'ouvre par un texte donné en bilingue, suivi par deux à trois textes à traduire en version, 
certains se trouvant en fin de chapitre. Ici aussi, l'élève dispose de divers exercices de traduction composés de phrases isolées. Il est à noter qu'il s'agit uniquement de versions.

Comme le «Latinissime », le «Magnard» et le «Latin Forum» interrompent régulièrement la suite des chapitres. Cependant, au lieu d'un chapitre de récapitulation toutes les cinq leçons, ils insèrent tous les deux chapitres dans le volume 1 , tous les trois chapitres dans les volumes 2 et 3 un chapitre différent, présentant des sujets culturels et historiques et appelé «Latin Magazine »; on y trouve notamment des textes d'auteurs en traduction.

\section{S'agit-il de texte(s) entièrement fabriqué(s), appuyé(s) sur des textes authentiques ou authentique(s) ? ${ }^{4}$}

Les « exercices gradués » du « Debeauvais » ne présentent pas de textes authentiques.

Le premier des deux volumes de «Godel» présente 39 textes: 19 textes, dont 4 inscriptions, authentiques, 15 adaptés, 5 fabriqués. Le second volume compte 44 textes : 39, dont 2 inscriptions, authentiques et 5 adaptés. La grande majorité des textes, qui illustrent des genres littéraires et textuels variés, est donc authentique. Il est précisé dans l'« Avertissement" que "la lecture de tous ces morceaux devra se faire sous l'active direction du maitre. Aucun d'entre eux ne devra être imposé comme devoir à domicile, ni donner lieu à un travail de contrôle » (Godel I, p.5).

À l'exception de ceux de la leçon 49 et de l'étape 10, les textes du «Latinissime » sont fabriqués, qu'il s'agisse de celui qui ouvre chaque leçon ou de ceux qui sont fournis dans le cadre des «étapes ». Cela correspond à un choix justifié dans les remarques liminaires sur la méthode: "Chaque texte a été créé ou adapté spécialement en fonction de la progression dans l'étude de la grammaire et du lexique, autour d'un centre d'intérêt adéquat. " (Latinissime, p.6)

30 À travers les trois volumes, le texte qui ouvre chaque chapitre du « Magnard » et est donné en bilingue est toujours un texte authentique. Les autres textes de chaque chapitre par contre sont fabriqués à partir de sources antiques; au fil des volumes, les séquences de mots reprises des textes authentiques sont de plus en plus nombreuses et de plus en plus longues. Le troisième volume présente en version presque deux fois plus de textes authentiques - 36 - que de textes adaptés - 19.

31 Les quatre textes que présente chaque chapitre des deux premiers volumes du « Latin Forum ", enfin, sont tous fabriqués; au fil des volumes, ils s'appuient également de plus en plus sur des textes authentiques. Les chapitres 1 des volumes 2 et 3 présentent chacun un long texte suivi fabriqué, narrant l'un les aventures d'Ulysse, l'autre les voyages de Lucius, inspirés par un texte authentique. Les chapitres 2 à 9 du troisième volume comptent un nombre plus élevé de texte, en moyenne six ; alors que quatre d'entre eux sont toujours fabriqués à partir de textes antiques, deux sont adaptés, voire authentiques. Le chapitre 10 contient 6 textes authentiques

\section{À quel moment du chapitre recourt-on au(x) texte(s) ?}

Comme il a déjà été dit, de petits textes apparaissent parfois au fil des «exercices gradués» du «Debeauvais». Il semble donc que l'emplacement qu'ils occupent à 
l'intérieur d'une séquence d'exercices est aléatoire ; par ailleurs, le manuel n'en propose pas une exploitation particulière.

L'emplacement des textes en fin des leçons du «Godel » est délibéré, comme les auteurs l'expliquent dans l'« Avertissement »: « les lectures sont destinées à récompenser les élèves de l'effort qu'ils auront fourni leçon après leçon » (Godel I, p.5).

Dans les trois autres manuels, «Latinissime ", "Magnard » et "Latin Forum », les premiers textes servent d'entrée dans le nouveau chapitre : dépourvus d'une traduction dans le «Latinissime » ou fournis avec une traduction dans le « Magnard » et le «Latin Forum », ils permettent des observations à partir desquelles les élèves induisent les sujets de grammaire, repris et schématisés ensuite par des explications grammaticales.

\section{Existe-t-il un lien entre les textes des diverses leçons?}

Tant pour le « Debeauvais » que pour le «Godel », la réponse à cette question est négative.

Les textes insérés dans les « exercices gradués » du «Debeauvais » traitent de sujets divers, dont quelques-uns relèvent de la culture romaine; cependant, aucun lien thématique plus étroit ne les relie.

En ce qui concerne le "Godel», les auteurs expliquent leur choix dans l'«Avertissement » : "il nous a paru bon de donner aux jeunes élèves une idée de la variété et de l'intérêt de la littérature latine » (Godel I, p.5).

Les textes qui ouvrent chaque leçon du «Latinissime » ont un lien entre eux : leur héros est un personnage imaginaire, un jeune Romain appelé Sextus, historiquement situé aux derniers temps de la République ; tout comme les élèves, Sextus grandit au fil du volume. Cette unité du personnage permet une variété de thèmes dans un contexte relativement bien défini.

9 Les textes proposés dans les brochures d'exercices genevoises n'entretiennent par contre aucun lien ni entre eux, ni avec les textes introductifs des leçons; ils traitent de sujets divers, allant de petites scénettes à des événements historiques ou des faits mythologiques.

Il a déjà été dit que les premiers textes de chaque chapitre du « Magnard » sont des textes authentiques; ils n'entretiennent aucun lien entre eux. Il en va autrement des textes suivants, qui narrent l'histoire de Rome depuis l'arrivée d'Énée à Carthage - chapitre 2 du premier volume - jusqu'au IV ème siècle de notre ère - chapitre 18 du troisième volume.

Tant le premier texte de chaque chapitre du « Latin Forum » que le suivant (volumes 1 et 2) ou les deux suivants (volume 3) sont consacrés à l'histoire de Rome, narrée depuis l'arrivée d'Énée à Carthage - chapitre 2 du volume 1 - jusqu'aux Antonins ( $2{ }^{\text {ème }}$ moitié du II ème siècle de notre ère) - «Prolongements» du volume 3. Ces textes sont donc étroitement reliés entre eux. Quant aux textes placés en fin de chapitres, ils sont reliés entre eux par une unité thématique: la vie quotidienne dans le premier volume, la mythologie et l'histoire dans le deuxième et le troisième volume; dans ce dernier apparait de plus une rubrique de textes adaptés ou authentiques intitulée « Faits divers ». 


\section{Existe-t-il un lien entre le(s) texte(s) et le(s) sujet(s) de grammaire de la leçon?}

latin. Le sujet de grammaire découvert grâce à ce texte fabriqué pour ainsi dire "sur mesure » et situé en ouverture de la leçon se trouve par la suite exercé par le biais de divers exercices, dont la traduction de phrases isolées. Quant aux brochures d'exercices genevoises, elles sont " conçues pour fournir aux maîtres enseignant avec le manuel "Salvete I " une batterie d'exercices visant à consolider l'acquisition de certaines notions, mais aussi une série d'activités permettant aux élèves débutants d'aborder le latin d'une manière aussi diversifiée que possible » (Salvete exercices $7^{\text {ème }}$ Fascicule 1, p.5). À la fin des exercices proposés pour chaque leçon se trouve, on l'a dit, un texte; composé pour exercer les sujets de grammaire de la leçon, ce texte entretient avec eux des liens d'application. découvrir le sujet de grammaire. Au fil du chapitre, les nouvelles acquisitions grammaticales et lexicales sont exercées par le biais de plusieurs textes, qui entretiennent donc un lien d'application avec les sujets grammaticaux. Comme il a été noté, ces textes ne sont pas authentiques; reliés entre eux par une unité thématique - l'histoire de Rome, ils n'ont aucun lien avec le texte de découverte.

Dans le "Latin Forum ", enfin, après la découverte inductive des sujets de grammaire à partir d'un texte fabriqué « sur mesure » et donné en bilingue, les nouvelles acquisitions sont exercées en deux étapes : d'abord à l'aide d'un ou deux texte(s) qui constitue(nt) la suite du texte de découverte, puis, dans une moindre mesure, par les textes donnés en fin de chapitre, reliés par une autre unité thématique et moins axés sur les sujets grammaticaux. Il convient de remarquer que le texte d'ouverture de chaque chapitre et le premier texte de version-dans le volume 3, les deux premiers textes de versions - illustrent la démarche de l'arche décrite par Rieunier (Rieunier I, p.198-199) :

Recherches en didactique des langues et des cultures, 13-3 | 2016 
pour schématiser, le premier induit une démarche d'induction, le(s) suivant(s) une démarche d'application que l'on pourrait qualifier aussi de déduction.

\section{Existe-t-il des liens nets entre le(s) texte(s) et le vocabulaire de la leçon?}

49 À l'exception du «Godel», tous les manuels proposent des textes créés ou adaptés précisément pour les leçons dans lesquelles ils apparaissent; ils contiennent donc, du moins partiellement, les mots qui composent le lexique de la leçon.

\section{Le manuel pose-t-il des questions de compréhension sur le(s) texte}

\section{(s)?}

Ni les « exercices gradués » du «Debeauvais », ni le «Godel » ne posent de questions de compréhension sur les textes proposés.

En ce qui concerne les «exercices gradués » du « Debeauvais », les textes y sont insérés dans la suite des exercices, comme il a déjà été remarqué. Pareillement aux exercices de traduction composés de phrases isolées, ces textes sont intitulés «version». Ce titre indique implicitement l'activité à accomplir - traduire du latin en français - et aucune autre consigne ne vient proposer une autre activité, telle l'interprétation.

Quant aux textes que le « Godel » place en fin de leçon, ils poursuivent un objectif double : d'une part récompenser l'élève des efforts fournis leçon après leçon ${ }^{5}$, d'autre part lui montrer la variété et l'intérêt de la littérature latine. Sans doute était-il prévu que le maitre qui devait superviser la lecture des élèves fournisse l'une ou l'autre explication et amène les élèves à la réflexion.

À nouveau, les trois autres manuels procèdent de façon semblable : tous trois interrogent les élèves sur le (premier) texte lu; tous trois poussent donc les élèves à s'impliquer activement dans la construction de leur savoir. Mais ici aussi, les trois manuels se distinguent par la suite.

Les auteurs du "Latinissime " focalisent en effet leurs questions sur la grammaire, comme ils l'expliquent dans les remarques liminaires à leur méthode: "des questions aident l'élève à organiser les paradigmes et à dégager le fonctionnement syntaxique par l'observation et la comparaison interne ou externe » (Latinissime p.6).

Les manuels «Magnard " et "Latin Forum » par contre joignent à leurs questions de découverte grammaticale des questions portant sur le sens du texte. Indépendamment du fait que dans le "Magnard ", elles concernent le texte authentique qui n'est pas réutilisé par la suite et que dans le " Latin Forum », elles portent sur le premier des deux (ou trois : volume 3 ) textes qui, chapitre après chapitre, brossent le tableau de l'histoire romaine, ces questions jouent un rôle important dans l'appréhension de la langue-culture qu'est le latin: en permettant d'établir un lien entre l'expression linguistique et le contenu du texte par le biais des deux opérations concomitantes que sont l'analyse de la langue et l'analyse du sens, elles inscrivent les faits de langue dans un contexte signifiant et permettent leur sédimentation. 


\section{Analyse} d'une bonne dizaine d'années pour ce qui est des exercices, présente certes également des exercices composés de phrases isolées généralement fabriquées; mais ces phrases fabriquées côtoient d'une part un nombre croissant de phrases authentiques, insérées dans les exercices et signalées par l'abréviation du nom de l'auteur donnée dans une parenthèse, et d'autre part des textes généralement authentiques placés après les exercices et dont la lecture recompensera les élèves de leurs efforts, comme il a déjà été dit. Malheureusement, les auteurs du «Godel» ne mentionnent pas les phrases authentiques dans leur "Avertissement ». On ne peut donc pas savoir si à leurs yeux, le caractère authentique de ces phrases les distinguait d'autres phrases, notamment par rapport à la latinité de l'expression et au contenu culturel. On ne peut que le supposer, puisqu'ils se sont donné la peine de les insérer et d'indiquer leur provenance.

60 S'ils présentent au début des textes fabriqués, les auteurs des deux manuels les plus récents, le « Magnard » et le « Latin Forum », cherchent par contre rapidement à recourir à des textes comportant de plus en plus d'éléments authentiques, conscients que la confrontation rapide avec la langue originale représente un enjeu cognitif important pour l'élève.

61 Le « Latinissime » fait ici un peu figure d'exception : inscrit dans l'évolution menant à une utilisation accrue du texte, il n'utilise pas des textes basés sur des sources antiques et il n'introduit des textes authentiques que très tard. Cela s'explique certainement par le souci des auteurs touchant la progression des apprentissages, comme le montre le passage déjà cité des remarques liminaires sur la méthode: «Chaque texte a été créé ou adapté spécialement en fonction de la progression dans l'étude de la grammaire et du lexique, autour d'un centre d'intérêt adéquat.» (Latinissime, p.6) Les auteurs des brochures d'exercices genevoises, désireux de pallier le manque de phrases isolées authentiques et de textes plus proches de l'expression latine, précisent ceci : «on trouvera dans ces six brochures de très nombreuses citations latines, glanées, triées et soumises à la sagacité des élèves genevois-es à la suite de la demande expresse des maitres de latin du canton. Il a paru [...] souhaitable aux maitres de proposer le plus tôt possible la lecture de citations et de textes d'auteurs, au besoin avec l'aide du maitre ou en recourant à des traductions pour certains 
passages. En mettant ainsi les élèves aux prises avec les textes anciens sous leur forme originelle, on les habitue à une certaine complexité de la pensée, de la langue et du style [...]. » (Avant-propos du dossier-maitre, p.2) Cette volonté a trouvé une concrétisation bien visible dans les quelques centaines de citations éparses à travers l'ensemble des fascicules.

Est-ce que quantité plus élevée de textes et mise en évidence accrue est synonyme d'un recours plus fréquent aux activités langagières et communicationnelles liées aux textes, à savoir, dans le cas du latin, lecture et médiation?

Pour répondre à cette question, il convient de tenir compte des trois points suivants. Premièrement, les manuels, même s'ils s'inscrivent dans un courant méthodologique, ne sont que des outils dans les mains des enseignants; il s'ensuit qu'ils reflètent certes les lignes générales du courant qu'ils illustrent, mais ne conditionnent pas intégralement le cours qu'ils contribuent à dispenser. Deuxièmement et par conséquent, la présence ou l'absence de certaines consignes ne signifie pas forcément que l'activité langagière en lien avec les consignes en question a ou n'a pas été réalisée. Finalement, les catégorisations en activités langagières et en modes de communication sont récentes; par conséquent, il serait vain de les chercher dans des manuels antérieurs. Ainsi, rien ne permet d'affirmer que les enseignants qui utilisaient les " exercices gradués» du "Debeauvais» ou le «Godel » n'étaient attentifs qu'à la grammaire et ne faisaient pas cas du contenu des phrases ou des textes, ni qu'ils ne pratiquaient jamais des activités relevant de la médiation, comme traduction, résumé, reformulation. On peut seulement constater que les consignes ne les y invitaient pas explicitement.

À la suite de ce constat, il convient de remarquer l'évolution quasi linéaire que dessine l'utilisation du texte au fil des cinq manuels. On est de fait passé de l'utilisation du texte comme application de règles de grammaire («exercices gradués » du «Debeauvais » et "Godel») à son utilisation de champ d'observation d'abord de nouveaux points de grammaire examinés dans leur contexte linguistique («Latinissime »), puis de nouveaux points de grammaire examinés dans un contexte sémantique («Magnard» et «Latin Forum»). Les deux derniers manuels font correspondre découverte grammaticale et construction du sens, ce qui montre bien l'importance d'un message pourvu d'un sens pour la compréhension d'une langue et son acquisition. Ainsi, le texte porteur de sens ${ }^{6}$ a remplacé le texte comme illustration de grammaire.

65 Les changements que l'on peut constater entre le "Debeauvais» d'une part et le «Magnard » et le «Latin Forum » de l'autre dénotent aussi deux soucis croissants, liés l'un à l'autre: celui de considérer le latin comme une langue-culture, à l'instar des langues modernes, avec la dimension de la communication orale en moins, et par conséquent celui d'imbriquer toujours davantage les unes aux autres les diverses connaissances attachées à l'étude d'une langue-culture, à savoir le fonctionnement lexical et grammatical de la langue tout comme les faits de culture.

\section{Conclusion}

L'analyse de cinq moyens d'enseignement de latin couvrant un certain laps de temps montre qu'ils sont passés d'un enseignement axé sur l'acquisition de la grammaire et du lexique à une approche privilégiant des activités langagières appuyées sur le travail sur le texte et autour de lui, les seules possibles en langues classiques parmi celles que définit le CECR. L'enseignement/apprentissage du latin a donc connu une évolution semblable à 
celui des langues modernes, qui, rappelons-le, tentait, dans la méthode indirecte, de ressembler le plus possible à l'enseignement des langues classiques: grands auteurs, exercices de thème et de version.

Pourquoi l'enseignement des langues anciennes a-t-il amorcé ce virage, à l'instar des langues modernes?

Est-ce pour trouver une réponse aux conséquences du changement de statut qu'ont vécu les langues classiques au sein de l'école ${ }^{7}$ ? De discipline obligatoire dans plusieurs filières, elles sont en effet passées au statut de discipline à option que les élèves peuvent abandonner - et abandonnent - à de nombreux moments. Aussi cherche-t-on à combler le fossé qui sépare les deux groupes de langues et à rapprocher l'enseignement des langues classiques de celui des langues modernes et donc de suivre le même "tournant communicatif » qu'elles: on n'attend plus que les élèves aient vu l'ensemble de la grammaire pour les convier à la lecture de textes, mais on les y conduit plus rapidement.

Le virage amorcé, bien que réel, n'est cependant pas très marqué: l'acquisition systématique de la grammaire continue à jouer un rôle très important, comme le montrent notamment deux points du "Latin Forum ", le plus récent des manuels : la progression est certes culturelle - l'histoire de Rome - mais surtout grammaticale; de plus, dans le cadre des exercices de manipulation et d'analyse grammaticale, il est demandé maintes fois de justifier la réponse par le recours à la règle grammaticale et non par le sens.

\section{BIBLIOGRAPHIE}

\section{Moyens d'enseignement}

\section{« Debeauvais » :}

Debeauvais, L. (1932 ; 1955). Grammaire latine. Paris : Belin et Lausanne : SPES.

Debeauvais, L. (1935). Cours de langue latine, à l'usage de l'enseignement secondaire, Vocabulaire. Paris : Belin.

Debeauvais, L. (1960). Cours de langue latine, exercices gradués, classe de $5^{e}$. Paris : Belin.

Debeauvais, L. (1962). Cours de langue latine, exercices gradués, classe de $6^{e}$. Paris : Belin.

« Godel » :

Godel, R. \& Chouet, M. (1966). Cours de Latin I. Genève : Georg.

Godel, R. \& Chouet, M. (1969). Cours de Latin II. Genève : Georg.

« Latinissime » :

Cousteix, J., Gaillard, J., Gouiran, G. \& Weinberg, R. (1992). Latinissime I. Lausanne : LEP

(adaptation romande de Cousteix, J., Gaillard, J., Gouiran, G. \& Weinberg, R. (1988), Salvete $4^{\text {ème }}$. Paris : Nathan). 
Cousteix, J., Gaillard, J., Gouiran, G. \& Weinberg, R (1993). Latinissime II. Lausanne : LEP (adaptation romande de Cousteix, J., Gaillard, J., Gouiran, G. \& Weinberg, R (1989), Salvete $4^{\text {ème }}$. Paris : Nathan) et II (Salvete $4^{\text {ème }}$ et $3^{\text {ème }}$, Lausanne - pour l'adaptation romande - 1992 et 1993).

\section{« Salvete » :}

Amstutz, Chr., Bader Lagomarsino, D. \& Chenaux, Cl. (2000). Salvete, Exercices $7^{e}$, Fascicule 1. Genève : DIP.

Amstutz, Chr., Bader Lagomarsino, D. \& Chenaux, Cl. (2000). Salvete, Exercices $7^{e}$, Fascicule 2. Genève : DIP.

Amstutz, Chr., Bader Lagomarsiono, D., Chappaz, J.-L, Chenaux, Cl. \& Vercellini, P. (2001). Salvete, Exercices $8^{e}$, Fascicule 1. Genève : DIP.

Amstutz, Chr., Bader Lagomarsiono, D., Chappaz, J.-L, Chenaux, Cl. \& Vercellini, P. (2001). Salvete, Exercices $8^{e}$, Fascicule 2. Genève : DIP.

Chappaz, J.-L, Chenaux, Cl. \& Vercellini, P. (2002). Salvete, Exercices $9^{e}$, Fascicule 1. Genève : DIP. Chappaz, J.-L, Chenaux, Cl. \& Vercellini, P. (2002). (2003). Salvete, Exercices $9^{e}$, Fascicule 2. Genève : DIP.

\section{« Magnard » :}

Gason, J., Lambert, A. \& Tréziny, H. (1997). Invitation au latin 5e. Paris : Magnard.

Gason, J., Lambert, A. \& Tréziny, H. (1998). Invitation au latin 4e. Paris : Magnard.

Gason, J., Lambert, A. \& Tréziny, H. (1999). Invitation au latin 3e. Paris : Magnard.

\section{« Latin Forum » :}

Agocs, M., Baud, M., Durussel, V., Kolde, A., Maréchaux, S., Rapin, A. (2012). Latin Forum $9^{e}$. Lausanne : DGEO.

Agocs, M., Baud, M., Durussel, V., Kolde, A., Maréchaux, S., Rapin, A. (2013). Latin Forum $10^{e}$. Lausanne : DGEO.

Agocs, M., Baud, M., Durussel, V., Kolde, A., Maréchaux, S., Rapin, A. (2014). Latin Forum 11e. Lausanne : DGEO.

\section{Bibliographie}

Armand, A. (1997). Didactique des langues classiques. Paris : Bertrand-Lacoste.

Conseil de l'Europe, 2001, Un cadre européen commun de référence pour les langues : apprendre, enseigner, évaluer. Paris : Didier.

Ko, M. (2000). Enseigner les langues classiques. Paris : Hachette.

Kolde, A. (2009). Langues anciennes et plurilinguisme, Babylonia 4, 67-69.

Marchi, G. (2015). Il metodo tradizionale : le ragioni di un fallimento, article en ligne consulté le 15.07.2016 : https://grecolatinovivo.wordpress.com/2015/06/14/metodo-tradizionale-ragioni-diun-fallimento/.

Martinez, P. (2008). La didactique des langues étrangères. Paris : PUF.

Rieunier, A. $\left(2001 ; 2007^{3}\right)$. Préparer un cours. Paris : ESF.

Waquet, F. (1998). Le latin ou l'empire d'un signe. XVI $I^{e} X X^{e}$ siècle. Paris : Albin Michel. 


\section{Sitographie}

Conseil de l'Europe, s.d., Division des Politiques linguistiques, www.coe.int/lang/fr, Les langues étrangères - vivantes et classiques.

\section{NOTES}

1. Conseil de l'Europe, Division des Politiques linguistiques, Les langues étrangères - vivantes et classiques. Sur ce texte, cf. notamment Kolde 2009.

2. Pour un historique de l'évolution de l'enseignement du latin en France, cf. Waquet et Ko (pp. 7-17).

3. Le «Debeauvais » couvre les années scolaires françaises de la $6^{\text {ème }}$ à la $3^{\text {ème }}$; les volumes se répartissent entre "Grammaire », "Grammaire complète », "Vocabulaire », "Exercices », « Exercices gradués » et « Textes latins ».

4. Par « texte entièrement fabriqué », on entend un texte rédigé par le ou les auteurs du manuel ; par «texte appuyé sur des textes authentiques », on entend un texte rédigé par le ou les auteurs du manuel, sur la base d'un ou de plusieurs textes d'auteurs antiques dont sont reprises des séquences de longueurs diverses, parfois légèrement modifiées par rapport au lexique ou à la syntaxe; par "texte authentique", on entend un texte tel qu'il a été rédigé par un auteur antique et n'ayant subi aucune intervention au niveau lexical ou syntaxique.

5. Cf. l'« Avertissement » Godel I, p.5: " les lectures sont destinées à récompenser les élèves de l'effort qu'ils auront fourni leçon après leçon». On ne connait pas le sentiment éprouvé par les élèves à la lecture de ces textes: on ne sait pas s'ils ont perçu cette lecture comme une récompense ou non et donc si l'objectif des auteurs a été atteint ou s'il est resté une projection.

6. Pour le recours au texte porteur de sens dans l'apprentissage des langues classiques, cf. Armand (1997) notamment p.100-101 et Ko (2000) passim. Armand (1997), p.100-101: «considérer le texte grec ou latin comme un texte porteur de sens, d'un sens qu'il faut construire grâce à des opérations qu'on demande expressément d'utiliser (...) : repérer, relier, justifier, commenter, en mobilisant des connaissances de tout ordre. (...) il faut avoir été habitué à formuler une hypothèse de sens à partir d'indices paratextuels, à partir d'une appréhension globale d'un sens fondée sur un premier repérage lexical ou stylistique grossier, puis à vérifier cette hypothèse grâce aux connaissances lexicales, morphologiques, syntaxiques, littéraires, historiques, en construisant le sens du détail et $d u$ tout dans de constants allers et retours faits d'anticipations et de vérifications, d'hypothèses et de prises de décision. C'est se révéler un lecteur compétent. "

7. Cf. Marchi (2015).

\section{RÉSUMÉS}

La contribution s'interroge sur la question de savoir si les manuels de latin connaissent une évolution semblable à ceux de langues modernes : remplacent-ils également l'objectif de l'acquisition grammaticale et lexicale par l'objectif communicationnel? Tant les manuels de latin que ceux de langues modernes proposent des activités sur et autour de textes; aussi, pour permettre la comparaison avec les manuels de langues modernes, la contribution propose-t-elle des réflexions sur la place que des manuels de latin de la 
seconde moitié du XX⿳亠丷⿵冂丶 activités proposées en lien avec les textes.

The contribution examines the question whether the Latin manuals develop in a similar way to those of modern languages: do they replace also the aim of grammatical and lexical acquisition by the communication objectives? Both Latin manuals and these of modern languages offer activities on and around texts; for comparison with the modern language textbooks, the contribution offers reflections on the place which Latin textbooks of the late twentieth century and early twenty-first century in western Switzerland grant to texts and the activities proposed in connection with the texts.

\section{INDEX}

Mots-clés : Latin, texte, moyens d'enseignement, manuels

Keywords : Latin, text, teaching books

\section{AUTEUR}

\section{ANTJE-MARIANNE KOLDE}

Haute École Pédagogique du canton de Vaud

Antje-Marianne Kolde est professeure en didactique du latin et du grec à la Haute École Pédagogique du canton de Vaud.

Domaines de recherche : didactique du latin et du grec ; lecture de textes grecs et latins ; plurilinguisme en cours de grec et de latin.

Courriel : antje-marianne.kolde[at]hepl.ch.

Adresse : Avenue de Cour 25 - CP, Haute École pédagogique, CH-1014 Lausanne. 